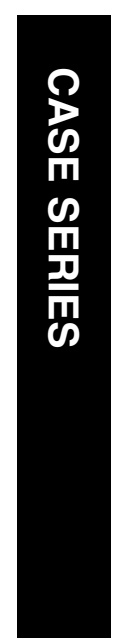

\title{
Familial central retinal vein occlusion
}

\begin{abstract}
Aim To report four cases of central retinal vein occlusion (CRVO) in a French family. Patients and methods Ophthalmological examination and medical work-up of seven members of the family.

Results There were four cases of CRVO in two consecutive generations. Three of them had CRVO in both eyes. Arterial hypertension was present in two, associated to glaucoma in one. Medical work-up did not reveal additional risk factors.

Conclusions We report a case of familial clustering of CRVO. Our cases combined to other cases reported in the literature provide arguments for the existence in some subjects of a genetic predisposition of CRVO.

Additional case series are however needed to confirm this hypothesis.
\end{abstract}

Ophthalmology of the Fondation Ophtalmologique Rothschild and of the Centre Hospitalier National des Quinze-Vingts, Université Pierre et Marie Curie, Paris, France

${ }^{2}$ Department of Clinical Genetic, Hospital Lariboisière, Assistance Publique-Hôpitaux de Paris, Université Paris 7, Paris, France

Correspondence: M Paques, Department of

Ophthalmology,

Fondation Ophtalmologique Rothschild,

25 rue Manin,

Paris 75019, France.

Tel: + 331480366 71;

Fax: + 33148036523 .

E-mail: michel.paques@

laposte.net

Received: 1 August 2006 Accepted in revised form: 30 October 2006

Published online:

15 December 2006

Competing interets: none

Financial disclosure: none

The authors have no proprietary interest in the present study
Eye (2008) 22, 308-310; doi:10.1038/sj.eye.6702672; published online 15 December 2006

Keywords: retinal vein occlusion

\section{Introduction}

Central retinal vein occlusion (CRVO)

is a potentially blinding disease, whose pathophysiology remains unknown.

We describe here a family with a high incidence of CRVO.

\section{Case reports}

\section{Case 1}

A 56-year-old woman (patient Ia) presented to one of us (SS) in February 1999 complaining of visual loss in the left eye. Her medical history comprised chronic headaches treated by oral propanolol. At initial examination, visual acuity (VA) was 20/20 OD, and 20/70 OS. Intraocular OS. Right fundus was normal. Left fundus pressure (IOP) was $16 \mathrm{mmHg}$ OD, $17 \mathrm{mmHg}$ showed perfused CRVO with macular oedema. A thorough medical and biological work-up (comprising dosage of protein C and S, antithrombin III, antiphospholipid antibodies, antinuclear antibodies, and plasma homocystein) did not reveal significant abnormalities. She was heterozygous for the C677T mutation of the methyltetrahydrofolate reductase (MTHFR) gene. One year later, CRVO occurred in the right eye. At that time, VA was 20/100 OD, and 20/50 OS. At the last visit in 2005, VA was 20/100 OD, 20/50 OS owing to bilateral macular oedema.

Case 2

Her brother (patient Id) developed CRVO in the right eye in July 1999 at age 72 . He had a history of high blood pressure and myocardial infarction, and received antihypertensive therapy and coumadin. VA was counting fingers OD, 20/20 OS. Anterior segment examination of the right eye revealed rubeosis iridis, and an IOP of $23 \mathrm{mmHg}$. IOP was $16 \mathrm{mmHg}$ OS. Right fundus examination revealed ischaemic CRVO. Left fundus was normal. Panretinal photocoagulation was subsequently performed in the right eye. Medical work-up (comprising dosage of erythrocyte sedimentation rate, fibrinogen, C, and S proteins, antiphospholipid antibodies, plasma homocystein, and of antithrombin III) was unremarkable. He was heterozygous for the C677T mutation of the MTHFR gene. In 2004, CRVO developed in the left eye. In 2005 he was seen by one of us (MP), and at that time VA was light perception OD, and $20 / 400$ OS.

Case 3

The son of the propositus (patient IIa) developed CRVO in the left eye in 1998 at the age of 27. He had an unremarkable medical history. At initial examination by one of us (SS), VA was 20/20 OD, and 20/100 OS. IOPs were normal. Fundus examination was normal OD, and showed perfused CRVO OS. Levels of 
Table 1 Reported cases of familial RVO

\begin{tabular}{|c|c|c|c|}
\hline Reference & $\begin{array}{l}\text { Age of onset in } \\
\text { generation } 1 \text { (years) }\end{array}$ & $\begin{array}{l}\text { Age of onset in } \\
\text { generation } 2\end{array}$ & Associated diseases/risk factors \\
\hline Castella and Othenin-Girard ${ }^{3}$ & 67 & $38 ; 41$ & $\begin{array}{l}\text { Hyperlipoproteinemia: } 3 \\
\text { HBP: } 3\end{array}$ \\
\hline Bhagat et al. ${ }^{1}$ & 46 & 17 & - \\
\hline Peris-Martinez et $a l^{2}$ & $38 ; 41$ & - & Prothrombin $20210 \mathrm{G} \rightarrow$ A mutation: 2 \\
\hline Puska et al. ${ }^{4}$ & $66 ; 41$; one unknown & 30 & Glaucoma: $4^{\text {a }} ; \mathrm{HBP}: 2$ \\
\hline Present report & 56; 76 ; one unknown & 29 & HBP: 2; glaucoma/HIOP: 1 \\
\hline
\end{tabular}

HBP: high blood pressure; HIOP: high intraocular pressure; RVO: retinal vein occlusion.

aWith myocilin Thr377Met mutation in three cases.

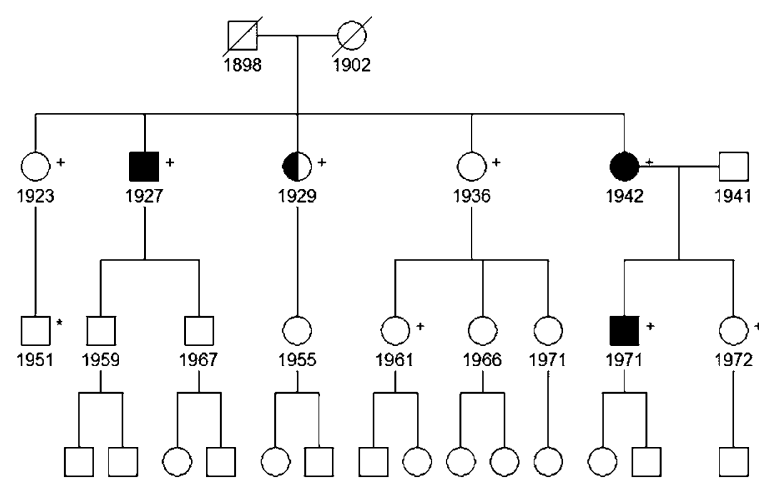

Figure 1 Pedigree of the family. Filled circles and squares: bilateral CRVO; half-filled circles and squares: unilateral CRVO; + indicate patients that have been examined by us.

proteins C and S, of antithrombin III, of antinuclear antibodies, of antiphospholipidin and of homocystein were within normal limits. Blood pressure was normal. In 2003, CRVO developed in the right eye. Iterative intravitreal injections of triamcinolone acetonide were performed in the right eye to treat macular oedema. At the last examination in June 2005, VA was 20/30 OD, and 20/160 OS.

Among the asymptomatic patients, one of the sisters of the propositus, patient Ic, was examined by one of us (MP) at the age of 72. She was under treatment for high blood pressure and high IOP. VA was 20/20 in both eyes. In the left eye, there was an opticociliary shunt suggestive of an asymptomatic episode of CRVO. The other members (Ib, Ie, and IIb) had normal fundi. Neither the parents nor the grandparents of the affected subjects were consanguineous. The cause of the death of the parents of case 1 are cancer for the mother, and heart disease for the father. None had known ocular disease. The family pedigree is shown in Figure 1.

\section{Comment}

In the literature, four reports of CRVO families, involving a total of 11 patients, have been published (Table 1). ${ }^{1-4}$
Such familial clustering of RVO is not per se a proof of the existence of a genetic predisposition to RVO. Indeed, given the little number of reported cases, these may be a coincidence. Moreover, most of these cases had significant risk factors for RVO such as arterial hypertension or glaucoma. ${ }^{5-9}$ However, an interesting finding is that offspring of affected patients had the disease at a younger age than their parents. Moreover, $46 \%$ had bilateral CRVO, and the mean age of onset was 45 years. Familial cases of CRVO cases appear thus to be more often bilateral $(P<0.01)$ and to affect younger patients $(P<0.05)$ than sporadic CRVO. ${ }^{5-7}$ Therefore, the figure that emerges is that familial CRVO may be a specific pathological entity, and thus deserves further studies. If the hypothesis of a genetic cause for these cases is correct, the mode of transmission cannot be determined with so few cases, and the collection of additional data from them and from other families is required.

\section{Acknowledgements}

We thank the Drs Claire Monin and Christian de Gennes for providing us clinical data concerning some of the cases showed here.

\section{References}

1 Bhagat N, Goldberg MF, Gascon P, Bell W, Haberman J, Zarbin MA. Central retinal vein occlusion: report of two familial cases. Eur J Ophthalmol 1999; 9: 181-195.

2 Peris-Martinez C, Dias-Llopis M, Menezo JL. Idiopathic central retinal vein occlusion in two siblings with the $20210 \mathrm{G} \rightarrow$ A variant. Arch Ophthalmol 2002; 120: 1752-1754.

3 Castella A, Othenin-Girard P. About three cases of familial central retinal vein occlusion associated with a familial hyperlipoproteinemia type II. Klin Monastilb Augenheheilk 1992; 200: 346-348.

4 Puska P, Lemmelä S, Kristo P, Sankila EM, Jarvela I. Penetrance and phenotype of the Thr377Met myocilin mutation in a large finnish family of juvenile-onset and 
adult-onset primary open angle glaucoma. Ophthalmic Genet 2005; 2: 17-23.

5 Mitchell P, Smith W, Chang A. Prevalence and associations of retinal vein occlusion in Australia. The Blue Mountains Eye Study. Arch Ophthalmol 1996; 114: 1243-1247.

6 The Central Vein Occlusion Study group. Natural history and clinical management of central retinal vein occlusion. Arch Ophthalmol 1997; 115: 486-491.

7 Sperduto RD, Hiller R, Chew E, Seigel D, Blair N, Burton TC et al. Risk factors for hemiretinal vein occlusion: comparison with risk factors for central and branch retinal vein occlusion: the eye disease case-control study. Ophthalmology 1998; 105: 765-771.

8 The Eye Disease Case-Control Study Group. Risk factors for central retinal vein occlusion. Arch Ophthalmol 1996; 114: 545-554.

9 McGimpsey SJ, Woodside JV, Bamford L, Gilchrist SE, Graydon R, McKeeman GC et al. Retinal vein occlusion, homocysteine, and methylene tetrahydrofolate reductase genotype. Invest Ophthal Vis Sci 2005; 46: 4712-4716. 